\title{
Existence and multiplicity results for a degenerate quasilinear elliptic system near resonance
}

\author{
Yu-Cheng An ${ }^{1,2}$, Xiong Lu ${ }^{1}$ and Hong-Min Suo ${ }^{1 *}$
}

\section{"Correspondence:}

gzmysxx88@sina.com

'School of Sciences, Guizhou Minzu

University, Guiyang, 550025,

P.R. China

Full list of author information is

available at the end of the article

\begin{abstract}
We establish existence and multiplicity results for weak solutions of a degenerate quasilinear elliptic system. By using Ekeland's variational principle, the mountain pass theorem and the saddle point theorem in critical point theory, we obtain the existence of one or three solutions for an elliptic system with Dirichlet boundary conditions under some restriction on $\lambda$. This kind of results was firstly obtained by Mawhin and Schmitt (Ann. Pol. Math. 51:241-248, 1990) for a semilinear two-point boundary value problem.
\end{abstract}

Keywords: degenerate elliptic system; Ekeland's variational principle; mountain pass theorem; saddle point theorem

\section{Introduction and main results}

In this paper, we consider the following degenerate quasilinear elliptic systems:

$$
\left\{\begin{array}{l}
-\nabla\left(h_{1}(x)|\nabla u|^{p-2} \nabla u\right)=\lambda a(x)|u|^{p-2} u+\lambda b(x)|u|^{\alpha}|v|^{\beta} v+F_{u}(x, u, v), \quad x \in \Omega, \\
-\nabla\left(h_{2}(x)|\nabla v|^{q-2} \nabla v\right)=\lambda d(x)|v|^{q-2} v+\lambda b(x)|u|^{\alpha}|v|^{\beta} u+F_{v}(x, u, v), \quad x \in \Omega, \\
\left.u\right|_{\partial \Omega}=\left.v\right|_{\partial \Omega}=0,
\end{array}\right.
$$

where $\Omega$ is a bounded and connected subset of $R^{N}(N \geq 2), \lambda$ is a nonnegative parameter, $F \in C^{1}\left(\bar{\Omega} \times R^{2}, R\right), \nabla F=\left(F_{u}, F_{v}\right)$ denotes the gradient of $F$ with respect to $(u, v) \in R^{2}$.

(f1) There exists a positive constant $C_{1}$ such that, for some $\theta \in(1, p), \tau \in(1, q)$,

$$
\begin{aligned}
& \left|F_{u}(x, u, v)\right| \leq C_{1}\left(1+|u|^{\theta-1}+|v|^{\tau(\theta-1) / \theta}\right), \\
& \left|F_{v}(x, u, v)\right| \leq C_{1}\left(1+|v|^{\tau-1}+|u|^{\theta(\tau-1) / \tau}\right),
\end{aligned}
$$

for all $(u, v) \in R^{2}$ and $x \in \Omega$.

(H) $p>1, q>1, \alpha \geq 0, \beta \geq 0$, such that

$$
\frac{\alpha+1}{p}+\frac{\beta+1}{q}=1
$$

The degeneracy of this system is considered in the sense that the measurable, nonnegative diffusion coefficients $h_{1}, h_{2}$ are allowed to vanish in $\Omega$ (as well as at the boundary $\partial \Omega$ )

\section{Springer}

2014 An et al: licensee Springer. This is an Open Access article distributed under the terms of the Creative Commons Attribution License (http://creativecommons.org/licenses/by/4.0), which permits unrestricted use, distribution, and reproduction in any medium, provided the original work is properly credited. 
and/or to blow up in $\bar{\Omega}$. The consideration of suitable assumptions on the diffusion coefficients will be based on [1], where the degenerate scalar equation was studied. We introduce the function space $(\mathrm{N})_{p}$ which consists of functions $h: \Omega \subset R^{N} \rightarrow R$, such that $h \in L^{1}(\Omega)$, $h^{-\frac{1}{p-1}} \in L^{1}(\Omega)$ and $h^{-s} \in L^{1}(\Omega)$, for some $p>1, s>\max \left\{\frac{N}{p}, \frac{1}{p-1}\right\}$ satisfying $p s \leq N(s+1)$.

Then for the weight functions $h_{1}, h_{2}$ we assume the following hypothesis:

(N) There exist functions $m_{1}$ satisfying condition $(\mathrm{N})_{p}$, for some $s_{p}$, and $m_{2}$ satisfying condition $(\mathrm{N})_{q}$, for some $s_{q}$, such that

$$
\frac{m_{1}(x)}{k_{1}} \leq h_{1}(x) \leq k_{1} m_{1}(x), \quad \frac{m_{2}(x)}{k_{2}} \leq h_{2}(x) \leq k_{2} m_{2}(x)
$$

a.e. in $\Omega$, for some constants $k_{1}>1$ and $k_{2}>1$.

The mathematical modeling of various physical processes, ranging from physics to biology, where spatial heterogeneity has a primary role, is reduced to nonlinear evolution equations with variable diffusion or dispersion. Note that problem (1) is closely related (see [1]) to the following system:

$$
\begin{cases}-\nabla\left(h_{1}(x, u, v)|\nabla u|^{p-2} \nabla u\right)=f(\lambda, x, u, v, \nabla u, \nabla v), & x \in \Omega, \\ -\nabla\left(h_{2}(x, u, v)|\nabla v|^{q-2} \nabla v\right)=g(\lambda, x, u, v, \nabla u, \nabla v), & x \in \Omega, \\ \left.u\right|_{\partial \Omega}=\left.v\right|_{\partial \Omega}=0 . & \end{cases}
$$

This system has successfully dealt with a variety of physical phenomena, such as the heat propagation in heterogeneous materials, the transport of electron temperature in a confined plasma, the propagation of varying amplitude waves in a nonlinear medium, the electromagnetic phenomena in nonhomogeneous superconductors, the dynamics of Josephson functions, and the spread of microorganisms. For more details as regards this type of system, see [2-4] and the references therein.

An example of the physical motivation of the assumptions $(\mathrm{N}),(\mathrm{N})_{p}$ may be found in [3]. These assumptions are related to the modeling of reaction diffusion processes in composite materials occupying a bounded domain $\Omega$, which at some points behave as perfect insulators. When at some points the medium is perfectly insulating, it is natural to assume that $h_{1}(x)$ and/or $h_{2}(x)$ vanish in $\Omega$. For more information, we refer the interesting reader to $[4]$ and the references therein.

For the perturbed problem, Mawhin and Schmitt [5] first considered the two-point boundary value problem

$$
-u^{\prime \prime}-\lambda u=f(x, u)+h(x), \quad u(0)=u(\pi)=0 .
$$

Under the assumption that $f$ is bounded and satisfies a sign condition, if the parameter $\lambda$ is sufficiently close to $\lambda_{1}$ from left, problem (3) has at least three solutions, if $\lambda_{1} \leq \lambda<\lambda_{2}$, problem (3) has at least one solution, where $\lambda_{1}, \lambda_{2}$ are the first, second eigenvalues of the corresponding linear problem. Ma et al. [6] considered the boundary value problem $\Delta u+\lambda u+f(x, u)=h(x)$ defined on a bounded open set $\Omega \subset R^{N}$, no matter whether the boundary conditions are Dirichlet or Neumann conditions, as the parameter $\lambda$ approaches $\lambda_{1}$ from left, there exist three solutions. Moreover, the existence of three solutions was obtained for the quasilinear problem in bounded domains as the parameter $\lambda$ approaches $\lambda_{1}$ from left in $[7,8]$, these results were extended to the perturbed $p$-Laplacian equation 
in $R^{N}$. Especially, the existence of three solutions has been extended to cooperative elliptic systems in [9]. Motivated by the above idea, we have the goal in this paper of extending these results to some degenerate quasilinear elliptic systems with the Dirichlet boundary conditions.

Next, we recall the basic results of the following eigenvalue problem (for details see [2]):

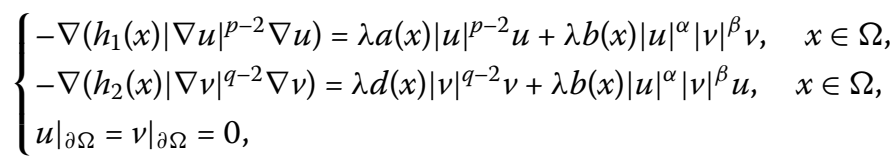

where $h_{1}, h_{2}$ satisfy (N), and the coefficient functions $a, d$, and $b$ satisfy conditions (A), (D), and (B), respectively (the conditions (A), (D), (B) see Section 2). Zographopoulos proved the theorem below.

Theorem 1.1 Let $\Omega$ be a bounded domain of $R^{N}(N \geq 2)$. Assume that hypotheses $(\mathrm{H})$ and $(\mathrm{N})$ are satisfied and the coefficient functions $a, d$ and $b$ satisfy conditions (A), (D) and (B), respectively. Denote by $L$ the set

$$
L=\left\{(u, v) \in Z: \frac{\alpha+1}{p} \int_{\Omega} a(x)|u|^{p} d x+\frac{\beta+1}{q} \int_{\Omega} d(x)|v|^{q} d x+\int_{\Omega} b(x)|u|^{\alpha}|v|^{\beta} u v d x=1\right\},
$$

where $Z=D_{0}^{1, p}\left(\Omega, h_{1}\right) \times D_{0}^{1, q}\left(\Omega, h_{2}\right)$ (we will determine in Section 2 what $Z$ means). Then the system (4) admits a positive principal eigenvalue $\lambda_{1}$, satisfying

$$
\lambda_{1}=\inf _{(u, v) \in L}\left\{\frac{\alpha+1}{p} \int_{\Omega} h_{1}(x)|\nabla u|^{p} d x+\frac{\beta+1}{q} \int_{\Omega} h_{2}(x)|\nabla v|^{q} d x\right\} .
$$

The associated normalized eigenfunction $\phi_{1}=\left(u_{1}, v_{1}\right)$ belongs to $Z$ and each component is nonnegative. In addition,

(i) the set of all eigenfunctions corresponding to the principal eigenvalue $\lambda_{1}$ forms a one-dimensional manifold $E_{1} \subset Z$, which is defined by

$$
E_{1}=\left\{\left(c_{1} u_{1}, c_{1}^{p / q} v_{1}\right): c_{1} \in R\right\}
$$

(ii) $\lambda_{1}$ is the only eigenvalue of (4) to which corresponds a componentwise nonnegative eigenfunction.

(iii) $\lambda_{1}$ is isolated in the following sense: there exists $\eta>0$, such that the interval $\left(0, \lambda_{1}+\eta\right)$ does not contain any other eigenvalue than $\lambda_{1}$.

Now we are ready to state our main results. For the related definitions we refer to Section 2.

Theorem 1.2 Let the hypotheses of Theorem 1.1 and (f1) be satisfied. In addition,

$$
\lim _{|t| \rightarrow \infty} F\left(x, t \phi_{1}\right)=+\infty
$$

uniformly in $x \in \Omega$. Then, for $\lambda<\lambda_{1}$ sufficiently close to $\lambda_{1}$, the system (1) has at least three solutions, where $\lambda_{1}>0$ is the first eigenvalue of the system (4). 
Theorem 1.3 Let the hypotheses of Theorem 1.1 and (f1) be satisfied. In addition,

$$
\lim _{|(u, v)| \rightarrow \infty}\left(\frac{1}{p} F_{u}(x, u, v)+\frac{1}{q} F_{v}(x, u, v)-F(x, u, v)\right)=\infty
$$

uniformly in $x \in \Omega$. Then for $\lambda \in\left(\lambda_{1}, \lambda_{2}\right)$, the system (1) has at least one solution (we will determine in Section 3 what $\lambda_{2}$ mean).

\section{Space and operator setting}

Let $h(x)$ be a nonnegative weight function in $\Omega$ which satisfies condition $(\mathrm{N})_{p}$. We consider the weighted Sobolev space $D_{0}^{1, p}(\Omega, h)$ to be defined as the closure of $C_{0}^{\infty}(\Omega)$ with respect to the norm

$$
\|u\|_{D_{0}^{1, p}(\Omega, h)}=\left(\int_{\Omega} h(x)|\nabla u|^{p} d x\right)^{1 / p} .
$$

The space $D_{0}^{1, p}(\Omega, h)$ is a reflexive Banach space. For a discussion of the space setting we refer to [1] and the references therein. Let

$$
p_{s}^{*}=\frac{N p s}{N(s+1)-p s} .
$$

Lemma 2.1 [1] Assume that $\Omega$ is a bounded domain in $R^{N}$ and the weight h satisfies $(\mathrm{N})_{p}$. Then the following embeddings hold:

(I) $D_{0}^{1, p}(\Omega, h) \hookrightarrow L^{p_{s}^{*}}(\Omega)$ continuously for $1<p_{s}^{*}<N$,

(II) $D_{0}^{1, p}(\Omega, h) \hookrightarrow L^{r}(\Omega)$ compactly for any $r \in\left[1, p_{s}^{*}\right)$.

In the sequel we denote by $p^{*}$ and $q^{*}$ the quantities $p_{s_{p}}^{*}$ and $q_{s q}^{*}$, respectively, where $s_{p}$ and $s_{q}$ are induced by condition $(\mathrm{N})$, recall that $h_{1}, h_{2}$ satisfy $(\mathrm{N})$. The assumptions concerning the coefficient functions of systems (1) and systems (4) are the following.

(A) $a \in L^{\frac{p^{*}}{p^{*}-p}}(\Omega)$ and either there exists $\Omega_{a}^{+} \subset \Omega$ of positive Lebesgue measure, i.e., $\left|\Omega_{a}^{+}\right|>0$, such that $a(x)>0$, for all $x \in \Omega_{a}^{+}$, neither $a(x) \equiv 0$, in $\Omega$.

(D) $d \in L^{\frac{q^{*}}{q^{*}-p}}(\Omega)$ and either there exists $\Omega_{d}^{+} \subset \Omega$ of positive Lebesgue measure, i.e., $\left|\Omega_{d}^{+}\right|>0$, such that $d(x)>0$, for all $x \in \Omega_{d}^{+}$, neither $d(x) \equiv 0$, in $\Omega$.

(B) $b(x) \geq 0$, a.e. in $\Omega, b \not \equiv 0$ and $b \in L^{\omega}(\Omega)$, where $\omega=\left(1-\frac{\alpha+1}{p^{*}}-\frac{\beta+1}{q^{*}}\right)^{-1}$.

The space setting for our problem is the product space $Z=D_{0}^{1, p}\left(\Omega, h_{1}\right) \times D_{0}^{1, q}\left(\Omega, h_{2}\right)$ equipped with the norm

$$
\|z\|=\|u\|_{D_{0}^{1, p}\left(\Omega, h_{1}\right)}+\|v\|_{D_{0}^{1, q}\left(\Omega, h_{2}\right)}, \quad z=(u, v) \in Z
$$

Observe that inequalities (2) in condition (N) imply that the functional spaces $D_{0}^{1, p}\left(\Omega, h_{1}\right) \times$ $D_{0}^{1, q}\left(\Omega, h_{2}\right)$ and $D_{0}^{1, p}\left(\Omega, m_{1}\right) \times D_{0}^{1, q}\left(\Omega, m_{2}\right)$ are equivalent. Next, let us introduce the functionals $I, J: Z \rightarrow R$ in the following way:

$$
\begin{aligned}
& I(u, v)=\frac{\alpha+1}{p} \int_{\Omega} h_{1}(x)|\nabla u|^{p} d x+\frac{\beta+1}{q} \int_{\Omega} h_{2}(x)|\nabla v|^{q} d x, \\
& J(u, v)=\frac{\alpha+1}{p} \int_{\Omega} a(x)|u|^{p} d x+\frac{\beta+1}{q} \int_{\Omega} d(x)|v|^{q} d x+\int_{\Omega} b(x)|u|^{\alpha}|v|^{\beta} u v d x .
\end{aligned}
$$


It is a standard procedure (see [10], Lemma 2.1) to prove the following properties of the functionals.

Lemma 2.2 The functionals $I, J$ are well defined. Moreover, $I$ is continuous and $J$ is compact.

Notation For simplicity we use the symbol $\|\cdot\|_{h_{1}}$ for the norm $\|\cdot\|_{D_{0}^{1, p}\left(\Omega, h_{1}\right)}$ and $\|\cdot\|_{h_{2}}$ for the norm $\|\cdot\|_{D_{0}^{1, q}\left(\Omega, h_{2}\right)}$.

\section{Proof of theorems}

Let $\Phi_{\lambda}: Z \rightarrow R$ be the functional defined by

$$
\Phi_{\lambda}(u, v)=I(u, v)-\lambda J(u, v)-N(u, v), \quad \forall(u, v) \in Z,
$$

where

$$
N(u, v)=\int_{\Omega} F(x, u, v) d x .
$$

Since the potential $F$ has sublinear growth, by a standard argument, it follows that $\Phi_{\lambda} \in$ $C^{1}(Z, R)$. In addition, $(u, v) \in Z$ is a weak solution of systems (1) if and only if $(u, v)$ is a critical point of $\Phi_{\lambda}$.

By (5) we can deduce that

$$
I(u, v) \geq \lambda_{1} J(u, v), \quad \forall(u, v) \in Z
$$

Let $V=\operatorname{span}\left\{\phi_{1}\right\}$ and $W=\left\{z \in Z:\left\langle J^{\prime}\left(\phi_{1}\right), z\right\rangle=0\right\}$, we have from the simplicity of $\lambda_{1}, Z=$ $V \oplus W$. Then, since $\lambda_{1}$ is also isolated, we have

$$
\lambda_{2}=\inf _{(u, v) \in W}\{I(u, v): J(u, v)=1\}
$$

which satisfies $\lambda_{1}<\lambda_{2}$. In addition,

$$
I(u, v) \geq \lambda_{2} J(u, v), \quad \forall(u, v) \in W .
$$

On the other hand, from the condition (f1) and by Young's inequality, for every fixed $\left(u_{0}, v_{0}\right) \in R^{2}$, it follows that

$$
\begin{aligned}
& \left|F\left(x, u_{0}, v_{0}\right)\right| \\
& =\left|\int_{0}^{1}\left(F_{u}\left(x, r u_{0}, r v_{0}\right) u_{0}+F_{v}\left(x, r u_{0}, r v_{0}\right) v_{0}\right) d r+F(x, 0,0)\right| \\
& \leq \int_{0}^{1}\left(\left|F_{u}\left(x, r u_{0}, r v_{0}\right)\right|\left|u_{0}\right|+\left|F_{v}\left(x, r u_{0}, r v_{0}\right)\right|\left|v_{0}\right|\right) d r+|F(x, 0,0)| \\
& \leq C_{1} \int_{0}^{1}\left(\left|u_{0}\right|+\left|u_{0}\right|^{\theta} r^{\theta-1}+\left|r v_{0}\right|^{\tau(\theta-1) / \theta}\left|u_{0}\right|+\left|v_{0}\right|+\left|v_{0}\right|^{\tau} r^{\tau-1}\right. \\
& \left.\quad+\left|r u_{0}\right|^{\theta(\tau-1) / \tau}\left|v_{0}\right|\right) d r+|F(x, 0,0)|
\end{aligned}
$$




$$
\begin{aligned}
\leq & |F(x, 0,0)|+C_{1}\left(\left|u_{0}\right|+\left|v_{0}\right|+\frac{1}{\theta}\left|u_{0}\right|^{\theta}+\frac{1}{\tau}\left|u_{0}\right|^{\tau}\right. \\
& \left.+\frac{\theta}{\theta+\tau(\theta-1)}\left|v_{0}\right|^{\tau(\theta-1) / \theta}\left|u_{0}\right|+\frac{\tau}{\tau+\theta(\tau-1)}\left|u_{0}\right|^{\theta(\tau-1) / \tau}\left|v_{0}\right|\right) \\
\leq & C_{2}\left(\left|u_{0}\right|+\left|v_{0}\right|+\left|u_{0}\right|^{\theta}+\left|v_{0}\right|^{\tau}+|F(x, 0,0)|\right),
\end{aligned}
$$

where $C_{2}$ is a positive constant independent to $\left(u_{0}, v_{0}\right)$, therefore, from Lemma 2.1, we have

$$
\begin{aligned}
\left|\int_{\Omega} F(x, u, v) d x\right| & \leq \int_{\Omega}|F(x, u, v)| d x \\
& \leq C_{2} \int_{\Omega}\left(|u|+|v|+|u|^{\theta}+|v|^{\tau}\right) d x+C_{2} \int_{\Omega}|F(x, 0,0)| d x \\
& \leq C_{2}\left(\|u\|_{L^{1}}+\|v\|_{L^{1}}+\|u\|_{L^{\theta}}^{\theta}+\|v\|_{L^{\tau}}^{\tau}\right)+C_{4} \\
& \leq C_{3}\left(\|u\|_{h_{1}}+\|v\|_{h_{2}}+\|u\|_{h_{1}}^{\theta}+\|v\|_{h_{2}}^{\tau}\right)+C_{4}
\end{aligned}
$$

for all $(u, v) \in Z$, where $C_{4}=C_{2} \int_{\Omega}|F(x, 0,0)| d x$ and $C_{3}$ is a positive constant.

Next, we will prove Theorem 1.2 by using Ekeland's variational principle and the mountain pass theorem and Theorem 1.3 by using the saddle point theorem.

Proof of Theorem 1.2 The proof will be divided into four steps.

Step 1. The functional $\Phi_{\lambda}$ is coercive in $Z, \Phi_{\lambda}$ is bounded from below on $W$ and there is a constant $M$, independent of $\lambda$, such that $\inf _{z \in W} \Phi_{\lambda}(z) \geq M$.

For $\lambda<\lambda_{1}$, from (8) and (11), we get

$$
\begin{aligned}
\Phi_{\lambda}(u, v) \geq & a_{1}\left(1-\frac{\lambda}{\lambda_{1}}\right)\left(\|u\|_{h_{1}}^{p}+\|v\|_{h_{2}}^{q}\right) \\
& -C_{3}\left(\|u\|_{h_{1}}+\|v\|_{h_{2}}+\|u\|_{h_{1}}^{\theta}+\|v\|_{h_{2}}^{\tau}\right)-C_{4},
\end{aligned}
$$

for all $(u, v) \in Z$, where $a_{1}=\min \{(1+\alpha) / p,(1+\beta) / q\}>0$, which shows that $\Phi_{\lambda}$ is coercive in $Z$.

Similarly, from (9) and (11), we obtain

$$
\begin{aligned}
\Phi_{\lambda}(u, v) \geq & a_{1}\left(1-\frac{\lambda_{1}}{\lambda_{2}}\right)\left(\|u\|_{h_{1}}^{p}+\|v\|_{h_{2}}^{q}\right) \\
& -C_{3}\left(\|u\|_{h_{1}}+\|v\|_{h_{2}}+\|u\|_{h_{1}}^{\theta}+\|v\|_{h_{2}}^{\tau}\right)-C_{4},
\end{aligned}
$$

for all $(u, v) \in W$. Hence $\Phi_{\lambda}$ is coercive in $W$ and $\Phi_{\lambda}$ is bounded from below on $W$. Moreover, there is a constant $M$, independent of $\lambda$, such that $\inf _{z \in W} \Phi_{\lambda}(z) \geq M$.

Step 2 . If the parameter $\lambda$ is sufficiently close to $\lambda_{1}$ from the left, we have $t^{-}<0<t^{+}$such that $\Phi_{\lambda}\left(t^{ \pm} \phi_{1}\right)<M$.

We choose $t^{+}>0$ sufficiently large, we get from (6) that $N\left(t^{+} \phi_{1}\right)>-M+1$, so that

$$
\begin{aligned}
\Phi_{\lambda}\left(t^{+} \phi_{1}\right) & <\left(1-\frac{\lambda}{\lambda_{1}}\right) I\left(t^{+} \phi_{1}\right)+M-1 \\
& \leq a_{2}\left(1-\frac{\lambda}{\lambda_{1}}\right)\left(\left\|t^{+} u_{1}\right\|_{h_{1}}^{p}+\left\|t^{+} \nu_{1}\right\|_{h_{2}}^{q}\right)+M-1,
\end{aligned}
$$


where $a_{2}=\max \{(1+\alpha) / p,(1+\beta) / q\}$. Then for $\lambda$ sufficiently close to $\lambda_{1}$ from the left, $\Phi_{\lambda}\left(t^{+} \phi_{1}\right)<M$. The same conclusion holds for a $t^{-}<0$.

Step 3. If $\lambda<\lambda_{1}$, the functional $\Phi_{\lambda}$ satisfies the (P.S.) condition. In addition, let

$$
\Theta^{ \pm}=\left\{z \in Z: z= \pm t \phi_{1}+\xi \text {, with } t>0 \text { and } \xi \in W\right\}
$$

$\Phi_{\lambda}$ satisfies $(P . S .)_{c, \Theta^{+}}$and $(P . S .)_{c, \Theta^{-}}$for all $c<M$.

On one hand, if $\left(u_{n}, v_{n}\right)$ be a (P.S.) sequence of $\Phi_{\lambda}$, that is,

$$
\Phi_{\lambda}\left(u_{n}, v_{n}\right) \rightarrow c \in R, \quad\left\|\Phi_{\lambda}^{\prime}\left(u_{n}, v_{n}\right)\right\|_{H^{*}} \rightarrow 0
$$

as $n \rightarrow \infty$. From Step 1 and (15), $\left(u_{n}, v_{n}\right)$ must be bounded in $Z$, that is, there is $K>0$ such that

$$
\left\|u_{n}\right\|_{h_{1}}+\left\|v_{n}\right\|_{h_{2}} \leq K
$$

for all $n \in N$. Thus, there is a subsequence of $\left(u_{n}, v_{n}\right)$, without any loss of generality still denoted by $\left(u_{n}, v_{n}\right)$, and $(u, v) \in Z$ such that $\left(u_{n}, v_{n}\right) \rightarrow(u, v)$ weakly in $Z,\left(u_{n}, v_{n}\right) \rightarrow(u, v)$ strongly in $L^{p}(\Omega) \times L^{q}(\Omega)$. Consequently, from (15) and (16), one has

$$
\lim _{n \rightarrow \infty}\left\langle\Phi_{\lambda}^{\prime}\left(u_{n}, v_{n}\right),\left(u_{n}-u, 0\right)\right\rangle=0 .
$$

From the condition (f1), Hölder's inequality, Lemma 2.1, and (16), it follows that

$$
\begin{aligned}
\left|\int_{\Omega} F_{u}\left(x, u_{n}, v_{n}\right)\left(u_{n}-u\right) d x\right| \leq & C_{1} \int_{\Omega}\left(1+\left|u_{n}\right|^{\theta-1}+\left|v_{n}\right|^{\tau(\theta-1) / \theta}\right)\left|u_{n}-u\right| d x \\
\leq & C_{1}\left(\left\|u_{n}\right\|_{L^{\theta}}^{\theta-1}+\left\|v_{n}\right\|_{L^{\tau}}^{\tau(\theta-1) / \theta}\right)\left\|u_{n}-u\right\|_{L^{\theta}} \\
& +C_{1}|\Omega|^{(p-1) / p}\left\|u_{n}-u\right\|_{L^{p}} \\
\leq & C_{1} S\left(\left\|u_{n}\right\|_{h_{1}}^{\theta-1}+\left\|v_{n}\right\|_{h_{2}}^{\tau(\theta-1) / \theta}\right)\left\|u_{n}-u\right\|_{L^{\theta}} \\
& +C_{1}|\Omega|^{(p-1) / p}\left\|u_{n}-u\right\|_{L^{p}} \\
\rightarrow & 0
\end{aligned}
$$

as $n \rightarrow \infty$, where $S$ is the embedding constant. Combining (17), (18), and Lemma 2.2, we get

$$
\lim _{n \rightarrow \infty} \int_{\Omega} h_{1}(x)\left|\nabla u_{n}\right|^{p-2} \nabla u_{n}\left(\nabla u_{n}-\nabla u\right) d x=0
$$

Similarly, we also obtain

$$
\lim _{n \rightarrow \infty} \int_{\Omega} h_{1}(x)|\nabla u|^{p-2} \nabla u\left(\nabla u_{n}-\nabla u\right) d x=0,
$$

hence, we conclude that

$$
\lim _{n \rightarrow \infty} \int_{\Omega} h_{1}(x)\left(\left|\nabla u_{n}\right|^{p-2} \nabla u_{n}-|\nabla u|^{p-2} \nabla u\right)\left(\nabla u_{n}-\nabla u\right) d x=0 .
$$


Therefore, using a well-known lemma, see for instance [11, Lemma 3.1] or [12, Lemma 4.1], we get $u_{n} \rightarrow u$ in $D_{0}^{1, p}\left(\Omega, h_{1}\right)$ as $n \rightarrow \infty$. Similarly, we get $v_{n} \rightarrow v$ in $D_{0}^{1, q}\left(\Omega, h_{2}\right)$ as $n \rightarrow \infty$, that is, $\left(u_{n}, v_{n}\right)$ has a convergent subsequence.

On the other hand, let $\left\{z_{n}\right\} \subset \Theta^{+}$satisfy $\Phi_{\lambda}\left(z_{n}\right) \rightarrow c<M$ and $\Phi_{\lambda}^{\prime}\left(z_{n}\right) \rightarrow 0$ as $n \rightarrow \infty$. Since $\Phi_{\lambda}$ is coercive and the potential $F$ satisfies the condition (f1), there is $z \in Z$ such that $z_{n} \rightarrow z$ strongly in $Z$. If $z \in \partial \Theta^{+}=W$, from the second conclusion of Step 1 , we get $\Phi_{\lambda}\left(z_{n}\right) \rightarrow c \geq M$, which is impossible. Hence $z \in \Theta^{+}$and $\Phi_{\lambda}$ satisfies the $(P . S .)_{c, \Theta^{+}}$condition. Similarly we see that $(P . S \text {. })_{c, \Theta^{-}}$holds for all $c<M$.

Step 4. Three solutions are obtained.

If $\lambda<\lambda_{1}$ is sufficiently close $\lambda_{1}$, from Step 1 and Step 2, we get $-\infty<\inf _{\Theta^{ \pm}} \Phi_{\lambda}<M$, which implies that $\Phi_{\lambda}$ is bounded below in $\Theta^{+}$. Consequently, from Ekeland's variational principle, there exists $\left\{z_{n}\right\} \subset \Theta^{+}$such that $\Phi_{\lambda}\left(z_{n}\right) \rightarrow \inf _{\Theta^{+}} \Phi_{\lambda}$ and $\Phi_{\lambda}^{\prime}\left(z_{n}\right) \rightarrow 0$ as $n \rightarrow \infty$. Since $\Phi_{\lambda}$ satisfies $(P . S .)_{c, \Theta^{+}}$for all $c<M$, there is $z^{+} \in \Theta^{+}$such that $\Phi_{\lambda}(z)=\inf _{\Theta^{+}} \Phi_{\lambda}$, that is, the infimum is attained in $\Theta^{+}$. A similar conclusion holds in $\Theta^{-}$. So $\Phi_{\lambda}$ has two distinct critical points, denoted by $z^{+}$and $z^{-}$.

To fix ideas, suppose that $\Phi_{\lambda}\left(z^{-}\right) \leq \Phi_{\lambda}\left(z^{+}\right)$, if $z^{+}$is not an isolated critical point, then $\Phi_{\lambda}$ has at least three solutions. Otherwise, putting

$$
\Psi(z)=\Phi_{\lambda}\left(z+z^{+}\right)-\Phi_{\lambda}\left(z^{+}\right), \quad e=z^{-}-z^{+},
$$

we have $\Psi(0)=0, \Psi(e) \leq 0$, and there exist $r>0, \rho>0$ such that $\Psi(z) \geq \rho$ if $\|z\|=r$. Then, since $\Phi_{\lambda}^{\prime}=\Psi^{\prime}$ and $\Psi$ also satisfies the (P.S.) condition, from the mountain pass theorem, the number

$$
c=\inf _{\gamma \in \Gamma \in[0,1]} \max _{t} \Phi_{\lambda}(\gamma(t)),
$$

where

$$
\Gamma=\left\{\gamma \in C([0,1], W): \gamma(0)=z^{+}, \gamma(1)=z^{-}\right\}
$$

is a critical value of $\Phi_{\lambda}$. Noting that all paths joining $z^{+}$to $z^{-}$pass through $W$, we have $c \geq M$. Therefore we have obtained a third critical point of $\Phi_{\lambda}$. The proof is now complete.

Proof of Theorem 1.3 The proof will be divided into two steps.

Step 1 (the $(P . S .)_{c}$ condition). Let $\left\{z_{n}=\left(u_{n}, v_{n}\right)\right\} \subset Z$ be such that there exists $c>0$ such that

$$
\left|\Phi_{\lambda}\left(z_{n}\right)\right| \leq c, \quad \forall n \in N
$$

and there exists a strictly decreasing sequence $\left\{\varepsilon_{n}\right\}_{n=1}^{\infty}$ with $\lim _{n \rightarrow \infty} \varepsilon_{n}=0$ such that

$$
\left|\left\langle\Phi_{\lambda}^{\prime}\left(z_{n}\right), z\right\rangle\right| \leq \varepsilon_{n}\|z\|, \quad \forall z \in Z
$$


We first prove that $\left\{z_{n}\right\}$ is bounded in $Z$, then by a standard argument, $\left\{z_{n}\right\}$ has a convergent subsequence. Suppose by contradiction that $\left\|z_{n}\right\| \rightarrow \infty$, we have

$$
\begin{aligned}
c+\varepsilon_{n}\left\|\left(u_{n}, v_{n}\right)\right\| & \geq\left|\Phi_{\lambda}\left(u_{n}, v_{n}\right)-\left\langle\Phi_{\lambda}^{\prime}\left(\left(u_{n}, v_{n}\right),\left(\frac{u_{n}}{p}, \frac{v_{n}}{q}\right)\right)\right\rangle\right| \\
& =\left|\int_{\Omega}\left(\frac{1}{p} F_{u}\left(x, u_{n}, v_{n}\right)+\frac{1}{q} F_{v}\left(x, u_{n}, v_{n}\right)-F\left(x, u_{n}, v_{n}\right)\right) d x\right| .
\end{aligned}
$$

Letting $n$ tend to infinity, we get

$$
\lim _{n \rightarrow \infty}\left|\int_{\Omega}\left(\frac{1}{p} F_{u}\left(x, u_{n}, v_{n}\right)+\frac{1}{q} F_{v}\left(x, u_{n}, v_{n}\right)-F\left(x, u_{n}, v_{n}\right)\right) d x\right|<C,
$$

where $C$ is a constant, which contradicts (7). Thus, $\left\{z_{n}\right\}$ is bounded. Since the nonlinearity $F$ satisfies the conditions (f1), by a standard argument, $\left\{z_{n}\right\}$ has a convergent subsequence.

Step 2 (the saddle point theorem). It is well known that the (P.S.) condition can be replaced by the $(P . S .)_{c}$ condition in the saddle point theorem of Rabinowitz (see [13, 14]). Then to conclude that $\Phi_{\lambda}$ has a critical point it suffices to show that

$$
\lim _{\|z\| \rightarrow \infty} \Phi_{\lambda}(z)=-\infty, \quad \lim _{\|w\| \rightarrow \infty} \Phi_{\lambda}(w)=+\infty
$$

where $z \in V=\operatorname{span}\left\{\phi_{1}\right\}$ and $w \in W$.

For $\lambda_{1}<\lambda<\lambda_{2}$, from (11), we get

$$
\begin{aligned}
\Phi_{\lambda}\left(t \phi_{1}\right)= & I\left(t \phi_{1}\right)-\lambda J\left(t \phi_{1}\right)-N\left(t \phi_{1}\right) \\
= & \left(1-\frac{\lambda}{\lambda_{1}}\right) I\left(t \phi_{1}\right)-N\left(t \phi_{1}\right) \\
\leq & a_{1}\left(1-\frac{\lambda}{\lambda_{1}}\right)\left(\left\|t u_{1}\right\|_{h_{1}}^{p}+\left\|t v_{1}\right\|_{h_{2}}^{q}\right) \\
& +C_{3}\left(\left\|t u_{1}\right\|_{h_{1}}+\left\|t v_{1}\right\|_{h_{2}}+\left\|t u_{1}\right\|_{h_{1}}^{\theta}+\left\|t v_{1}\right\|_{h_{2}}^{\tau}\right)+C_{4} .
\end{aligned}
$$

Since $\theta \in(1, p), \tau \in(1, q)$, the first part of (21) holds.

For $\lambda_{1}<\lambda<\lambda_{2}$ and for any $w \in W$, from (13), we obtain the second statement of (21). The proof is now complete.

Competing interests

The authors declare that they have no competing interests.

Authors' contributions

All authors contributed equally to the writing of this paper. All authors read and approved the final manuscript.

Author details

'School of Sciences, Guizhou Minzu University, Guiyang, 550025, P.R. China. ${ }^{2}$ School of Sciences, Bijie University, Bijie, 551700, P.R. China.

\section{Acknowledgements}

This work was supported by the Science and Technology Foundation of Guizhou Province (No. LKB[2012]19; No. [2013]2141). 


\section{References}

1. Drábek, P, Kufner, A, Nicolosi, F: Quasilinear Elliptic Equations with Degenerations and Singularities. de Gruyter, Berlin (1997)

2. Zographopoulos, NB: On the principal eigenvalue of degenerate quasilinear elliptic systems. Math. Nachr. 281, 1351-1365 (2008)

3. Dautray, R, Lions, JL: Mathematical Analysis and Numerical Methods for Science and Technology. Springer, Berlin (1990)

4. Karachalios, NI, Zographopoulos, NB: On the dynamics of a degenerate parabolic equation: global bifurcation of stationary states and convergence. Calc. Var. Partial Differ. Equ. 25, 361-393 (2006)

5. Mawhin, J, Schmitt, K: Nonlinear eigenvalue problems with the parameter near resonance. Ann. Pol. Math. 51, 241-248 (1990)

6. Ma, TF, Ramost, M, Sanchez, L: Multiple solutions for a class of nonlinear boundary value problem near resonance: a variational approach. Nonlinear Anal. 30, 3301-3311 (1997)

7. Ma, TF, Pelicer, ML: Perturbations near resonance for the $p$-Laplacian in $R^{N}$. Abstr. Appl. Anal. 7, 323-334 (2002)

8. Ma, TF, Ramost, M: Three solutions of a quasilinear elliptic problem near resonance. Math. Slovaca 47, 451-457 (1997)

9. Ou, ZQ, Tang, CL: Existence and multiplicity results for some elliptic systems at resonance. Nonlinear Anal. 71, 2660-2666 (2009)

10. Drábek, P, Stavrakakis, NM, Zographopoulos, NB: Multiple nonsemitrivial solutions for quasilinear elliptic systems. Differ. Integral Equ. 16, 1519-1532 (2003)

11. Brock, F, Iturriaga, J, Sánchez, J, Ubilla, P: Existence of positive solution for $p$-Laplacian problems with weight. Commun. Pure Appl. Anal. 5, 941-952 (2006)

12. Ghoussoub, N, Yuan, C: Multiple solutions for quasi-linear PDEs involving the critical Sobolev and Hardy exponents. Trans. Am. Math. Soc. 352, 5703-5743 (2000)

13. Bartolo, P, Benci, V, Fortunato, D: Abstract critical point theorems and applications to some nonlinear problems with strong resonance at infinity. Nonlinear Anal. 7, 981-1012 (1983)

14. Rabinowitz, PH: Minimax Methods in Critical Point Theory with Applications to Differential Equations. CBMS (1986)

doi:10.1186/s13661-014-0184-5

Cite this article as: An et al.: Existence and multiplicity results for a degenerate quasilinear elliptic system near resonance. Boundary Value Problems 2014 2014:184.

\section{Submit your manuscript to a SpringerOpen ${ }^{\circ}$ journal and benefit from:}

- Convenient online submission

- Rigorous peer review

- Immediate publication on acceptance

Open access: articles freely available online

- High visibility within the field

- Retaining the copyright to your article 